\title{
Utilisation de la diffusion centrale anomale pour l'étude des séparations de phases d'alliages ternaires
}

\author{
O. LYON et J.P. SIMON*
}

\author{
Laboratoire pour l'Utilisation du Rayonnement Electromagnétique, CNRS, Bât. 209D, 91405 Orsay, \\ France \\ * Laboratoire de Thermodynamique et Physico-Chimie Métallurgiques, CNRS et INPG, BP. 75, \\ 38402 Saint Martin d'Hères, France
}

\begin{abstract}
RESUME
La diffusion centrale est une méthode de choix pour l'étude de la cinétique de séparation de phases. Néanmoins les mesures de rayons X classiques, ne détectant que les fluctuations de densité électronique, ne permettent pas de différentier la contribution des différents types d'atomes dans un alliage ternaire. La diffusion anomale, par effet de résonance entre l'absorption $\mathrm{X}$ et la diffusion élastique au voisinage d'un seuil d'absorption d'un élément, modifie le facteur de diffusion atomique de cet élément et, de ce fait, est sélective. L'étude de la séparation de phases d'alliages ternaires sera l'occasion d'illustrer différentes potentialités de la diffusion centrale anomale (ASAXS): discriminer entre différentes interprétations, déterminer la composition des phases, enfin plus généralement connaître la répartition spatiale des éléments grâce au formalisme des facteurs de structure partiels.
\end{abstract}

\begin{abstract}
Small Angle Scattering is a powerfull tool to study phase separation in alloys. Nevertheless, standard X rays, sensitive to electronic density fluctuations, are unable to differentiate the different elements in a ternary alloy. The Anomalous Scattering, through a resonance between absorption and elastic scattering, changes the atomic scattering factor of the given element and is therefore element selective. On exemples borrowed to the study of phase separation in ternary alloys, several aspects of Small Angle Anomalous X-ray Scattering will be emphazised: discrimination between several interpretations, determination of phase compositions or, more generally, of the spatial pattern for the three elements through the formalism of Partial Structure Factors.
\end{abstract}

\section{I - INTRODUCTION}

La séparation de phases, considérée comme générique des transitions de phase avec paramètre d'ordre conservé, a fait l'objet d'une intense activité théorique. Du point de vue appliqué, sa connaissance a aidé à un meilleur contrôle des alliages à durcissement structural. L'article précédent a présenté le cas d'un certain nombre d'alliages binaires considérés comme prototypes, mais un certain nombre d'alliages ternaires ( $\mathrm{Cu} \mathrm{Ni} \mathrm{Fe,} \mathrm{Cu} \mathrm{Ni} \mathrm{Sn,} \mathrm{Fe} \mathrm{Cr} \mathrm{Co}, \mathrm{Al} \mathrm{Zn} \mathrm{Ag...)} \mathrm{ont} \mathrm{aussi} \mathrm{été} \mathrm{étudiés} \mathrm{comme} \mathrm{tels.} \mathrm{Cependant,} \mathrm{la}$ présence du troisième élément offre un degré supplémentaire de liberté au système et des chemins de décomposition et cinétiques inattendus sont possibles. En effet si la thermodynamique permet de prévoir les diagrammes de phase d'équilibre, tels que celui représenté sur la figure 1, elle ne donne aucun renseignement sur le chemin suivi, ni sur la cinétique de décomposition. Par exemple, pour l'alliage initialement homogène de composition $c_{i o}$, quelle est l'évolution après trempe vers les compositions d'équilibre de l'état biphasé à basse température $\left(c_{i m}\right.$ et $\left.c_{i p}\right)$ ? Les études antérieures de diffusion centrale ont toutes supposé que la décomposition suivait la conode (trait plein de la Fig. 1), mais si l'on se réfère aux modèles linéaires de décomposition spinodale, la décomposition suivrait lors des premiers stades la direction du plus grand vecteur propre de la matrice d'amplification des ondes de concentration (flèches de la Fig. 1). 


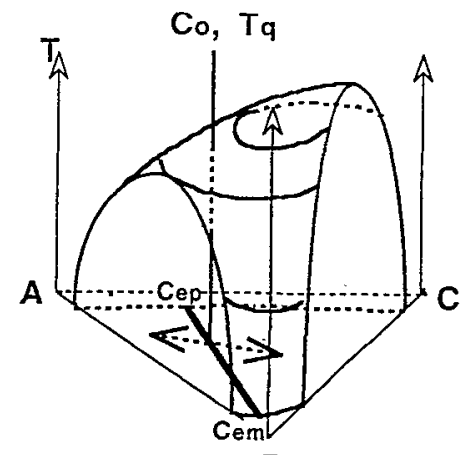

B.
Fig.1: Vue en perspective d'un diagramme de phase ternaire A B C. La lacune de miscibilité est la surface supportée par les deux lacunes binaires "AB" et "BC". Une conode est indiquée en trait gras et une direction spinodale par une flèche.

L'anomal, grâce à l'effet de résonance entre la diffusion élastique et l'absorption au voisinage d'un seuil d'absorption d'un élément, permet sélectivement de modifier le facteur de diffusion atomique correspondant, et donc d'étiqueter un type d'atome particulier.

Différentes facettes du problème de la séparation de phases d'alliages ternaires permettront d'illustrer certaines potentialités de la diffusion centrale anomale:

- aider au choix entre différentes interprétations

- préciser la composition des phases quand des interfaces nets se sont développés.

- analyser en détail la distribution des éléments dans des alliages ternaires à séparation de phase grâce au formalisme des facteurs de structure partiels.

Ce papier commencera par une description des procédures expérimentales et méthodes d'analyse.

\section{II - MÉTHODES EXPÉRIMENTALES ET ANALYSE:}

II-1 Facteur de diffusion atomique:

Le facteur de diffusion des rayons $X$ s'exprime sous la forme: $f=f_{0}+f^{\prime}(E)+i f^{\prime \prime}(E)$. Aux petits angles, $f_{o}$ est constant, égal au numéro atomique $Z$. La partie imaginaire $f^{\prime \prime}(E)$ est proportionnelle à l'absorption et subit donc un saut au niveau d'un seuil d'absorption de l'élément. La résonance entre la diffusion élastique et l'absorption au voisinage d'un seuil conduit au terme $f^{\prime}(E)$ qui peut atteindre -8 électrons au niveau des seuils K et -30 électrons au niveau des seuils $\mathrm{L}_{\text {III. }}$. En dessous d'un seuil d'absorption, $\mathrm{f}^{\prime \prime}<<\mathrm{f}^{\prime}$ et $f^{\prime \prime}$ et $f^{\prime}$ ne comportent pas d'oscillations dépendantes de l'état structural de l'échantillon (oscillations EXAFS). Les valeurs tabulées de $\mathrm{f}^{\prime}$ et $\mathrm{f}^{\prime \prime}$ à partir du formalisme de Cromer et Libermann sont donc utilisables tant que la distance relative au seuil $\left(E-E_{s}\right) / E_{s}$ est bien supérieure a la bande passante du monochromateur et à la largeur du seuil. En pratique, des valeurs effectives de $f$ et $\mathrm{f}^{\prime \prime}$ sont mesurées en dessous de $310^{-3}$, la gamme d'énergie optimale se situant en général entre $510^{-2}$ et $10^{-3} / 1,2 /$.
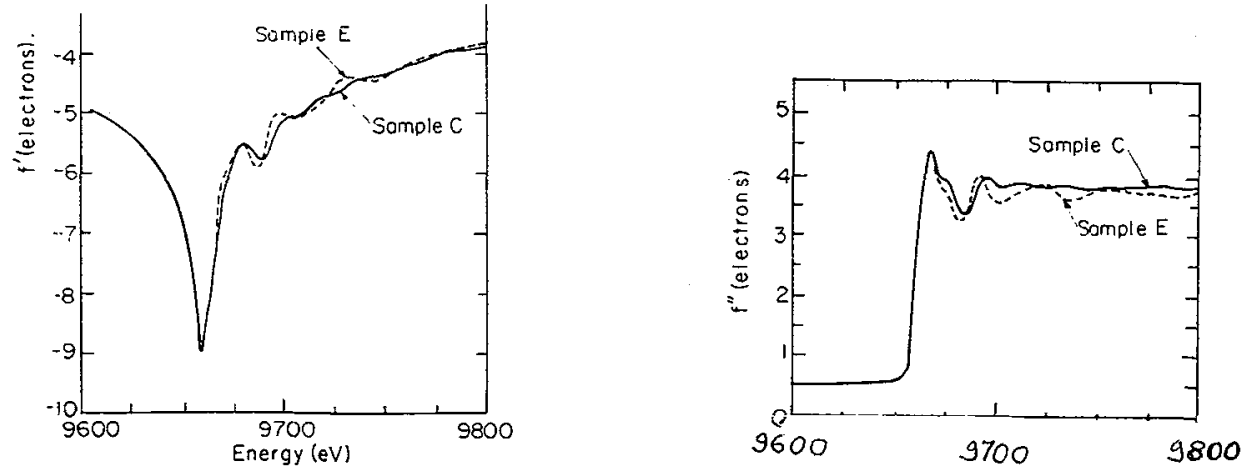

Fig 2: Facteurs anomaux de diffusion atomique $\mathrm{f}^{\prime}$ et $\mathrm{f}^{\prime \prime}$ du $\mathrm{Zn}$ au seuil $\mathrm{K}$ dans $\mathrm{Al} 14 \% \mathrm{Zn} 4 \% \mathrm{Ag}(\mathrm{C})$ et dans $\mathrm{Al} 4 \% \mathrm{Zn} 14 \% \mathrm{Ag}(\mathrm{E}) / 3 /$ 


\section{II-2 Conditions expérimentales:}

L'ASAXS couple un appareillage de diffusion aux petits angles avec un monochromateur installe sur une source blanche, sur une ligne de lumière synchrotron. La configuration simple de la ligne de D22 de LURE /4/ est présentée sur la figure 3. On réalise la résolution en énergie par un monochromateur à deux cristaux à sortie fixe et par une collimation grâce aux fentes $S_{1}$. La résolution est de l'ordre de $210^{-4}$ pour les $\mathrm{Si}_{311}$ et de $10^{-3}$ pour les $\mathrm{Ge}_{111}$ aux longueurs d'onde (aux énergies) où le flux de DCI permet les mesures $(\sim 4-12 \mathrm{keV})$. La caméra de SAXS consiste en un assemblage modulaire sous vide, comprenant fentes, moniteurs, porte-échantillons et puits, ce qui minimise le bruit de fond parasite et permet de modifier la géométrie à volonté. Les mesures ont été réalisées avec des détecteurs à gaz linéaires ou bidimensionnels. Comme ces détecteurs n'ont qu'une sélection en énergie grossière $(-20 \%)$, il faut éviter la fluorescence de l'échantillon et les mesures sont donc réalisées à des énergies en dessous du seuil choisi.

M1

\section{M2}

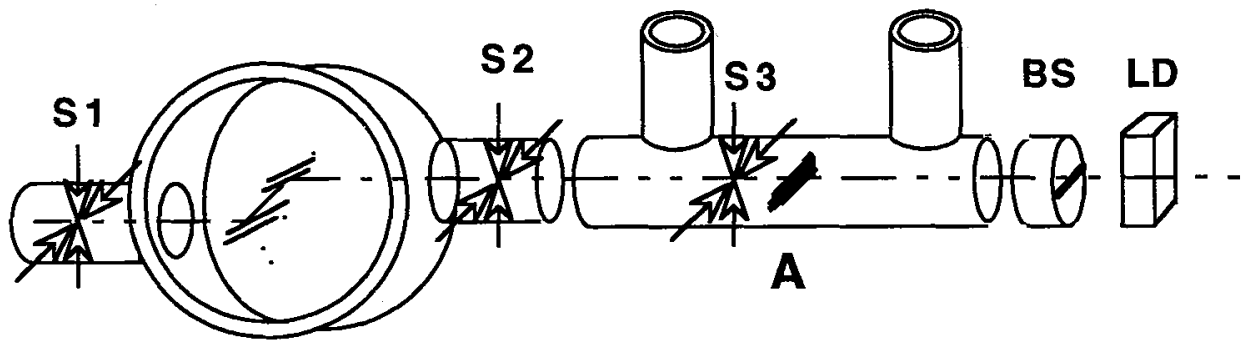

\section{Mono}

Fig.3 : Montage de diffusion centrale anomale : $\mathrm{S}$ fentes, $\mathrm{M}$ moniteurs, DL détecteur linéaire.

II-3 Analyse de l'ASAXS en terme de facteurs de structure partiels (PSFs):

Pour pouvoir accéder à l'occupation des sites par les différents types d'atome, il faut être en mesure de déterminer les fonctions de corrélation de paires par leurs transformées de Fourier "les facteurs de structure partiels". L'analyse suit le même formalisme que celui utilisé pour déterminer la structure des amorphes binaires si ce n'est que les mesures sont à petits angles.

Le facteur de structure partiel "PSF" entre atomes i et $\mathrm{j}$ s'écrit :

$$
\operatorname{Sij}(q)=N_{i}(q) N_{j}^{*}(q)=\int N_{i}(r) N_{j}(r) \exp \left[i q\left(r_{i}-r_{j}\right)\right] d^{3} r
$$

$\mathrm{N}_{\mathbf{i}}(\mathrm{r})$ est la densité d'occupation par l'élément $\mathrm{i}$, les atomes $\mathrm{i}$ contribuant pour une amplitude partielle

$F_{i}(q)=f_{i} \int_{v} N_{i}(r) \exp (i q r) d^{3} r$.

La section différentielle $d \sigma / d \Omega$ est donnée par le carré de l'amplitude $\mathrm{F}(\mathrm{q})$ totale, $\mathrm{F}(\mathrm{q}) \mathrm{F}(\mathrm{q})^{*}$, :

$$
\frac{\mathrm{d} \sigma}{\mathrm{d} \Omega}=\sum_{\mathrm{j}=1, \mathrm{~N}} \mathrm{f}_{\mathrm{i}} \mathrm{f}^{*} S_{\mathrm{ij}}
$$

Dans un système à $\mathrm{N}$ éléments, il y a $\mathrm{N}(\mathrm{N}+1) / 2$ PSFs, qui sont indépendants dans un système amorphe et qui sur un réseau, sont liés par les relations de conservation du soluté. Il n'en reste donc que $\mathrm{N}(\mathrm{N}-1) / 2$ PSFs indépendants dans les cristaux.

Comme, jusqu'à présent, on n'a pu résoudre que des problèmes à trois PSFs indépendants, l'équation (2) développée devient alors :

$$
\frac{d \sigma}{d \Omega}=F_{1} F_{1} * S_{11}(q)+\left(F_{1} F_{2} *+F_{1} * F_{2}\right) S_{12}(q)+F_{2} F_{2} * S_{22}(q)
$$

où les $F$ s sont des contrastes vis à vis du troisième élément o pris comme référence $\left(F_{1}=f_{1}-f_{0}, F_{2}=f_{2}-f_{0}\right)$. Il faut au moins trois mesures indépendantes afin d'inverser le système linéaire (3). 
Classiquement, ce type d'études faisait appel à la substitution isotopique en neutrons, mais, cette technique s'est avérée inadaptée parce qu'il est incertain d'arriver à saisir le même niveau d'avancement de la démixtion, i.e. des états structuraux identiques, dans les trois échantillons. Un tel essai pour l'étude de la démixtion de l'Al $\mathrm{Zn} \mathrm{Ag}$ avec substitution isotopique d'Ag avait montré l'inadéquation des neutrons pour ce type d'études: des PSFs non physiques, en particulier des valeurs négatives de $\mathrm{S}_{\mathrm{AgAg}}$, ont été trouvées et la simulation a indiqué qu'une différence de $1 \%$ de taille des précipités dans les trois échantillons à isotopes différents suffisait pour calculer des PSFs non physiques $15.6 \%$.

L'alternative est la diffusion centrale anomale (ASAXS) qui se fait sur le mếme échantillon.

L'extraction des PSFs nécessite donc l'inversion du système linéaire (3), soit en principe trois mesures avec des contrastes $F_{i}$ différents. En pratique, les variations anomales sont si faibles que le système est singulier avec des mesures d'ASAXS au voisinage d'un seul seuil, et il faut impérativement des mesures aux seuils de deux éléments. On appelle conditionnement, l'amplification des erreurs expérimentales qu'entraîne l'inversion du système d'équations (3). Il est typiquement de quelques dizaines. Mais la possibilite de surdimensionner le système (grâce à la source continuement ajustable de synchrotron) contrebalance partiellement ce mauvais conditionnement et différentes méthodologies ont été développées pour en tirer parti /1, 6-8/.

II-4 Modèle à deux phases:

Lorsqu'il n'est pas possible de mesurer deux seuils, ou si l'on a la certitude d'avoir des particules, on utilise le modèle à 2 phases: il suppose que les domaines de compositions différentes ont des interfaces abrupts et sont homogènes en composition. Pour être bref, on appelera la phase majoritaire matrice $m$ et la phase minoritaire particules ou précipités p. (Cette dénomination peut être abusive dans le cas où les deux fractions volumiques sont équivalentes entraînant une morphologie bipercolée). Si l'on soustrait la contribution du Laue (diffusion diffuse constante provenant du désordre, de l'incohérence spatiale), la diffusion à petits angles résiduelle (au sens de Gerold /9/) est le produit d'un contraste entre les phases $\mathrm{p}$ et $m$ et d'un facteur de structure géométrique $S_{\phi}(q)$, transformée de Fourier des corrélations de paires $r_{p}-(r+x) p$, (fonction de Patterson)

$$
\frac{d \sigma}{d \Omega}=\left(\rho_{p}-\rho_{m}\right)^{2} S_{\phi}(q)
$$

En l'absence d'effet de taille entre phases $m$ et $p\left(V=V_{p}=V_{m}\right)$, la densité électronique s'écrit : $\rho_{j}(j=m, p)=\frac{1}{V} \sum_{i} C_{i j} f_{i}$.

Après avoir tenu compte de la conservation des concentrations sur le réseau et après avoir particularisé un des types d'atomes "o" (par exemple l'élément non anomal) le contraste prend la forme suivante :

$$
\left(\rho_{p}-\rho_{m}\right)=\frac{1}{V} \sum_{i=1,2}\left(C_{i p}-C_{i m}\right)\left(f_{i}-f_{o}\right)
$$

et à partir des équations (6) et (7) on obtient la proportionnalité suivante avec le facteur de structure géométrique $S_{\phi}(q)$ :

$$
S_{i j}(q)=\left(C_{i p}-C_{i m}\right)\left(C_{j p}-C_{j m}\right) S_{\phi}(q)
$$

Le premier critère d'existence d'un modèle à deux phases est bien évidemment que les différents profils anomaux aient la même forme puisqu'ils doivent être proportionnels à $S_{\phi}(q)$. Mais ce critère n'est pas suffisant, car l'intensité des PSFs doit être compatible avec l'équation (6). Le cas du Cu Ni Fe (cf § III3) est précisement un contre exemple.

Lorsque l'équation (4) est vérifiée, elle l'est quelque soit $\mathrm{q}$, et l'utilisation des intensités intégrées améliore la précision de l'analyse. L'intensité diffusée en dessous du seuil de l'élément "1" en l'absence de fluorescence est telle que

$$
\sqrt{d \sigma / d \Omega}=\left(C_{1 p}-C_{1 m}\right)\left(f_{1}(E)-f_{0}\right)+\left(C_{2 p}-C_{2 m}\right)\left(f_{2}-f_{0}\right)
$$

De cette relation linéaire avec $f_{1}(E)$ on en déduit $X=\left(C_{2 p}-C_{2 m}\right) /\left(C_{1 p}-C_{1 m}\right)$, la pente du chemin de décomposition. 


\section{III - SÉPARATION DE PHASE DANS LES ALLIAGES TERNAIRES:}

Le système binaire $\mathrm{Fe} \mathrm{Cr}$ est modèle pour la vérification des théories de décomposition. Les études neutroniques ( voir le papier précédent de F. Bley) sont limitées en dessous de $520^{\circ} \mathrm{C}$ du fait de l'apparition de la phase $\sigma$ alors que la température critique extrapolée est voisine de $650^{\circ} \mathrm{C}$. On ne peut donc accéder qu'aux stades peu avancés de démixtion. L'addition de Co inhibe la précipitation de $\sigma$ et la cinétique de décomposition a ainsi pu être mesurée par sonde atomique $/ 10 \%$.

Pour le système CuNi, la température critique $\left(400^{\circ} \mathrm{C}\right)$ est si basse, i.e. les mobilités atomiques étaient si réduites qu'il est exclu d'y étudier la décomposition thermique. Dans ce système, l'addition de Fe déplace vers les hautes températures la lacune de miscibilité et la cinétique de décomposition a ainsi pu être mesurée par diffusion des neutrons aux petits angles /11/. Le système $\mathrm{Cu} \mathrm{Ni} F e$ a donc aussi retenu notre attention, car sous certains aspects il est similaire à $\mathrm{Fe} \mathrm{Cr} \mathrm{Co}$ (même type de diagramme de phase, fraction volumique de l'ordre de $50 \%$ pour les deux phases, conduisant à une morphologie bipercolée), mais il s'y développe une anisotropie de décomposition.

\section{III-1 Evaluation critique de la nature des objets contribuant à la diffusion centrale:}

En plus de la bosse de diffusion associée à la séparation de phase (telle la Fig. 5 ), on observe dans la plupart des alliages et même des verres métalliques une décroissance rapide de l'intensité aux tous petits angles: cette décroissance est en général attribuée à de gros défauts parasites, tels que précipités intergranulaires, rugosités de surface, dislocations...Dans le cas de la diffusion centrale des neutrons sur $\mathrm{Cu} \mathrm{Ni} \mathrm{Fe,} \mathrm{Poerschke} \mathrm{et} \mathrm{al.} \mathrm{/12/} \mathrm{l'avaient} \mathrm{néanmoins} \mathrm{interprété} \mathrm{en} \mathrm{terme} \mathrm{de} \mathrm{démixtion} \mathrm{avec} \mathrm{une}$ précipitation bimodale. Ne comprenant pas quel mécanisme pouvait conduire à une répartition en taille présentant deux maxima, nous avons tout d'abord voulu nous assurer que la diffusion au niveau de la queue et celle au niveau de la bosse provenaient bien du même type d"'objets diffuseurs". Contrairement aux variation anomales mesurées au niveau de la bosse, d'un facteur deux tant au voisinage du seuil du Fe que de celui du $\mathrm{Ni}$, la variation anomale de la queue de diffusion (mesurée entre 0.04 et $0.2 \mathrm{~nm}^{-1}$ ) est très faible $(<10 \%)$ (Fig 4). Quantitativement elle se comporte comme le carré de la densité électronique moyenne de l'alliage: il s'agit donc du contraste d'un défaut superficiel, tel que rugosité de surface ou pores et non celui d'autres précipitations de grande taille $/ 13 /$.

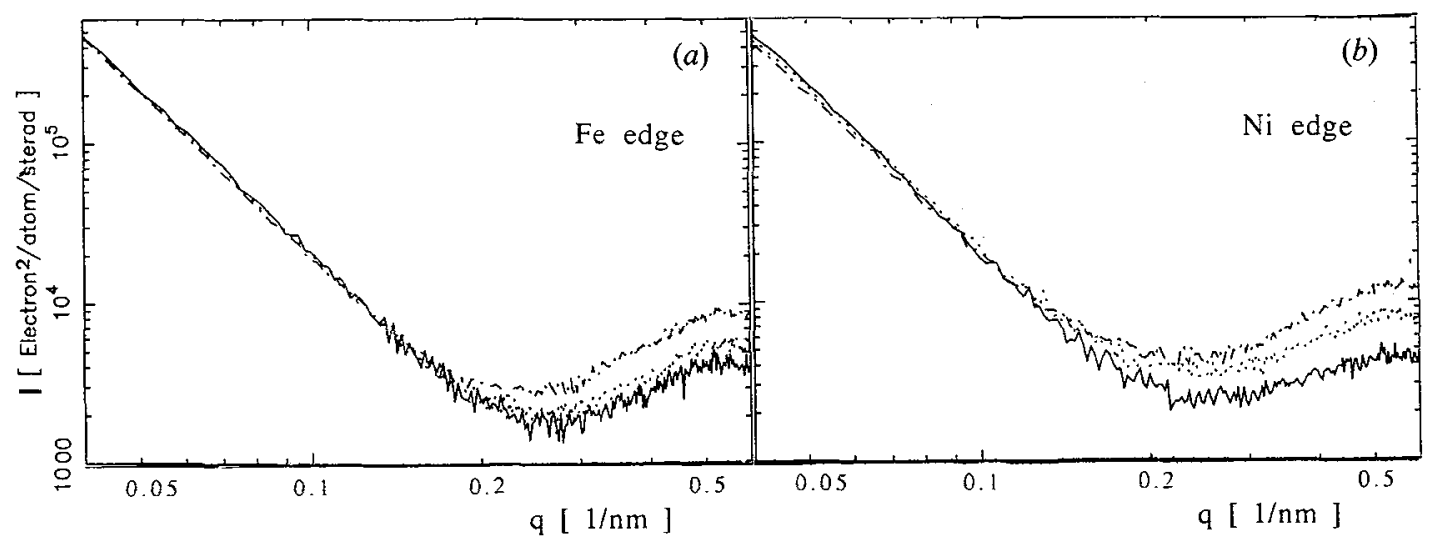

Fig.4: Profils de diffusion centrale à différentes énergies dans $\mathrm{Cu}_{0.425} \mathrm{Ni}_{0.425} \mathrm{Fe}_{0.15}$ vieilli $56 \mathrm{~h}$ à $773 \mathrm{~K}$. a) près du seuil $\mathrm{K}_{\mathrm{Fe}}(7112 \mathrm{eV})$ : traits pleins $(6996 \mathrm{eV})$, pointiliés $(7056 \mathrm{eV})$, pointillés longscourts $(7104 \mathrm{eV})$; b) près du seuil $\mathrm{K}_{\mathrm{Ni}}(8333 \mathrm{eV})$ : trait plein $(8150 \mathrm{eV})$, pointille $(8295 \mathrm{eV})$, pointille long-court $(8322 \mathrm{eV})$; le signal du à la démixtion est apparent au delà de $0,2 \mathrm{~nm}^{-1}$. 


\section{III-2 Etude du $\mathrm{Fe} \mathrm{Cr} \mathrm{Co}$ : une démixtion pseudo binaire}

Pour différents vieillissements à $566^{\circ} \mathrm{C}$, des mesures d'ASAXS aux deux seuils $\mathrm{Cr}$ et $\mathrm{Fe}$ (Fig 5) ont permis d'extraire les facteurs de structure partiels (Fig 6) $/ 2 /$.

L'équation (3) s'écrit dans le cas du Fe $\mathrm{Cr} \mathrm{Co}$ :

$$
\frac{\mathrm{d} \sigma}{\mathrm{d} \Omega}(\mathrm{q}, \mathrm{E})=\left(\mathrm{F}_{\mathrm{Cr}}\right)^{2} \mathrm{~S}_{\mathrm{CrCr}}(\mathrm{q})+\left(\mathrm{F}_{\mathrm{Fe}} \mathrm{F}_{\mathrm{Cr}} *+\mathrm{F}_{\mathrm{Fe}} \mathrm{F}_{\mathrm{Cr}}\right) \mathrm{S}_{\mathrm{FeCr}}(\mathrm{q})+\left(\mathrm{F}_{\mathrm{Fe}}\right)^{2} \mathrm{~S}_{\mathrm{FeFe}}(\mathrm{q})
$$

$\operatorname{avec} \mathrm{F}_{\mathrm{Fe}}=\mathrm{f}_{\mathrm{Fe}}-\mathrm{f}_{\mathrm{Co}}$ et $\mathrm{F}_{\mathrm{Cr}}=\mathrm{f}_{\mathrm{Cr}}-\mathrm{f}_{\mathrm{Co}}$

Les trois PSFs indépendants homoéléments ont des formes similaires, avec des intensités augmentant de $\mathrm{S}_{\mathrm{CoCo}}$ à $\mathrm{S}_{\mathrm{FeFe}}$, à $\mathrm{S}_{\mathrm{CrCr}}$. Comme les profils d'ASAXS et les PSFs semblaient proportionnels, la première hypothèse fut un modèle à deux phases. Les PSFs sont parfaitement compatibles avec une structure biphasée dont la pente $\left(\mathrm{C}_{\mathrm{Fe} \mathrm{p}}-\mathrm{C}_{\mathrm{Fe} \mathrm{m}}\right) /\left(\mathrm{C}_{\mathrm{Cr}} \mathrm{p}^{-} \mathrm{C}_{\mathrm{Crm}}\right)$ est égale à la conode, confirmant les résultats de microscopie ionique de champs /10/ (Fig 7). Le système se comportant comme un alliage pseudobinaire $\mathrm{Fe}-\mathrm{Co}$ et $\mathrm{Cr}$, les résultats cinétiques peuvent être comparés avec les modèles de démixtion binaire : la coalescence suit un comportement universel et la longueur pertinente varie en $t^{0,25}$, régime souvent observé pour la coalescence des systèmes bipercolés (F. Bley et al.).

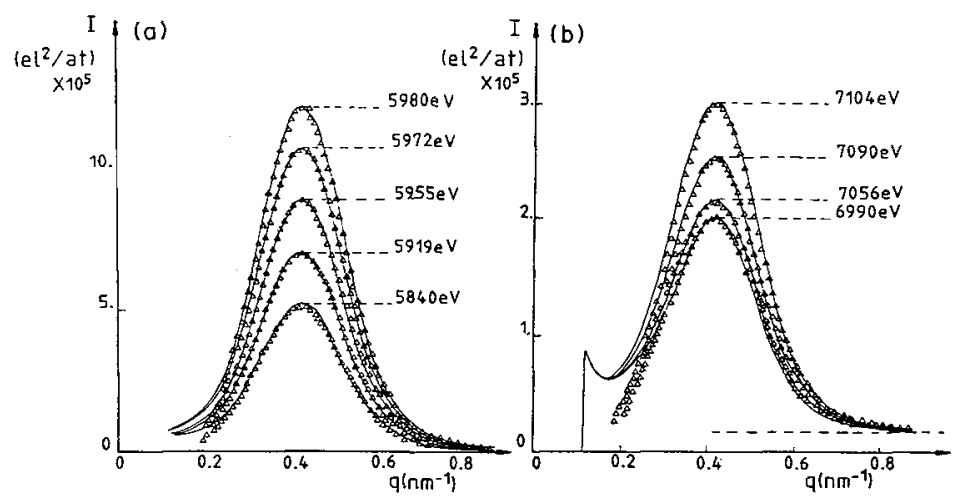

Fig 5 Intensité expérimentale anomale corrigée d'un $\mathrm{Fe} 29 \% \mathrm{Cr} 12 \% \mathrm{Co}$ vieilli $64 \mathrm{~h}$ à $566^{\circ} \mathrm{C}$ (a) près du seuil $\mathrm{K}_{\mathrm{Cr}}$, (b) près du seuil $\mathrm{K}_{\mathrm{Fe}}$; Les triangles correspondent aux intensités reconstruites à partir des "meilleurs" PSF, ceux de la figure 6.

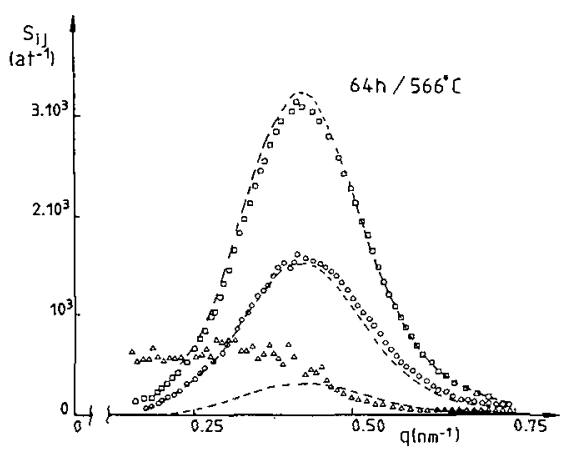

Fig 6 Facteurs de structures partiels (PSFs) d'un Fe $29 \% \mathrm{Cr} 12 \% \mathrm{Co}$ vieilli $64 \mathrm{~h}$ à $566^{\circ} \mathrm{C}$ :

a $\mathrm{S}_{\mathrm{CrC}}$, o $\mathrm{S} F \mathrm{Fe}, \Delta \mathrm{S}_{\mathrm{CoCo}}\left(=2 \mathrm{~S}_{\mathrm{CrFe}}+\mathrm{S}_{\mathrm{FeFe}}+\right.$ $\mathrm{S}_{\mathrm{CrCr}}$ ). Les pointillés correspondent à l'approximation du modèle à deux phases.

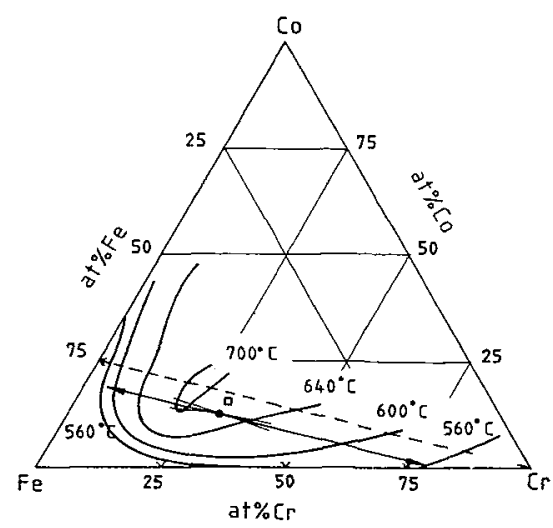

Fig 7 Comparaison de la lacune de miscibilité d'équilibre et des conodes déduites des mesures cinétiques: en trait plein, nos résultats d'ASAXS $/ 2 /$ en pointillé, ceux de sonde atomique $/ 10 /$. 


\section{III-3 Demixtion du $\mathrm{Cu} \mathrm{Ni} F e$ : des profils de concentration différents pour les trois éléments}

L'alliage polycristallin, $\mathrm{Cu}_{0.425} \mathrm{Ni}_{0.425} \mathrm{Fe}_{0.15}$, a été étudié par ASAXS pour différents traitements thermiques entre $460^{\circ} \mathrm{C}$ et $550^{\circ} \mathrm{C} / 14,15 /$.

Après soustraction de la queue de diffusion à petits angles, il subsiste la bosse centrée à $0.5 \mathrm{~nm}^{-1}$ pour $56 \mathrm{~h}$ à $500^{\circ} \mathrm{C}$. L'intensité de cette bosse augmente de $85 \%$ près du seuil $\mathrm{K}_{\mathrm{Fe}}$ (entre $6875 \mathrm{et} 7104 \mathrm{eV}$ ) et de $115 \%$ près du seuil $\mathrm{K}_{\mathrm{Ni}}$ (entre 8150 et $8322 \mathrm{eV}$ ). Les trois PSFs indépendants ainsi que $\mathrm{S}_{\mathrm{CuCu}}\left(=2 \mathrm{~S}_{\mathrm{NiFe}}\right.$ $+\mathrm{S}_{\mathrm{FeFe}}+\mathrm{S}_{\mathrm{NiNi}}$ ) sont représentés sur la Fig. 8: tous les $\mathrm{S}_{\mathrm{ii}}(\mathrm{q})$ sont positifs comme ils doivent être. Ils ont des formes similaires avec des intensités augmentant de $\mathrm{S}_{\mathrm{FeFe}}$ à $\mathrm{S}_{\mathrm{NiNi}}$, à $\mathrm{S}_{\mathrm{CuCu}}$. Comme les profils d'ASAXS et les PSFs sont presque proportionnels, la première hypothèse fut un modèle à deux phases. Le chemin de décomposition a une pente, $\left(\mathrm{C}_{\mathrm{Ni}} \mathrm{p}-\mathrm{C}_{\mathrm{Nim}}\right) /\left(\mathrm{C}_{\mathrm{Fe}}-\mathrm{C}_{\mathrm{Fe}} \mathrm{m}\right)$, égale à $2.0 \pm 0.2$. Des domaines enrichis en $\mathrm{Ni}$ et $\mathrm{Fe}$ alternent avec des domaines riches en $\mathrm{Cu}$, en accord avec des mesures magnétiques et avec les profils de concentration obtenus en analyse par sonde ionique (APM with FIM) 111. Les valeurs du rapport $X$ dans d'autres alliages $\mathrm{Cu}$ Ni Fe sont respectivement de $2.0 \pm 0.2$ pour $\mathrm{Cu}_{0.69} \mathrm{Ni}_{0.24} \mathrm{Fe}_{0.07}, 2.8 \pm 0.3$ pour $\mathrm{Cu}_{0.5} \mathrm{Ni}_{0.41} \mathrm{Fe}_{0.09}$ et de 1.5 pour $\mathrm{Cu}_{0.33} \mathrm{Ni}_{0.42} \mathrm{Fe}_{0.23}$. Par comparaison avec le diagramme de phase calculé, la décomposition suit la conode.

Mais, contrairement au cas du $\mathrm{Fe} \mathrm{Cr} \mathrm{Co,} \mathrm{il} \mathrm{apparait} \mathrm{que} \mathrm{ce} \mathrm{modèle} \mathrm{à} \mathrm{deux} \mathrm{phases} \mathrm{n'est} \mathrm{qu'une} \mathrm{première}$ approximation grossière. En effet.de l'équation (6) du modèle à 2 phases, on déduit immédiatement l'identité :

$$
\mathrm{S}_{\mathrm{FeNi}}{ }^{2}=\mathrm{S}_{\mathrm{NiNi}} \cdot \mathrm{S}_{\mathrm{FeFe}}
$$

qui n'est par obéie. $S_{\mathrm{FeNi}}$ est en effet plus petit que $\mathrm{S}_{\mathrm{NiNi}}$ et que $\mathrm{S}_{\mathrm{FeFe}}$ (Fig. 8). Si on introduit un terme supplémentaire $S_{\varepsilon}$, il a été montré que les partiels devaient s'exprimer comme une somme de deux termes $S_{\phi}, S_{\varepsilon}$ (Fig. 8):

$$
S_{i i}(q)=X_{i}^{2} S_{\phi}(q)+Y_{i}^{2} S_{\varepsilon}(q)
$$
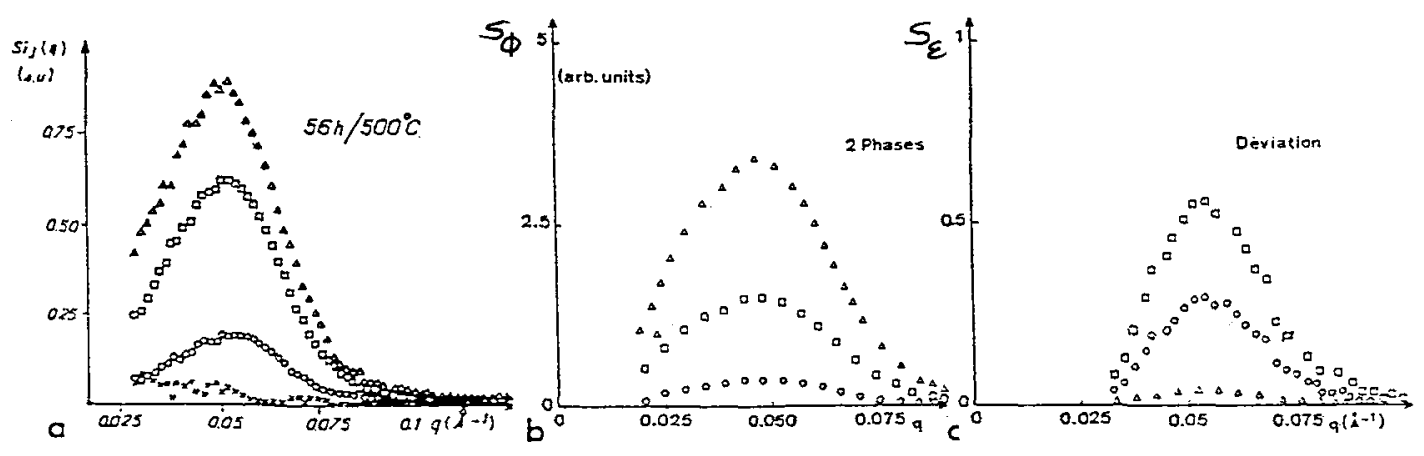

Fig. 8: Facteurs de Structure Partiels d'un échantillon $\mathrm{Cu}_{0.425} \mathrm{Ni}_{0.425} \mathrm{Fe}_{0.15}$ polycristallin /15/:

(a) $\Delta S_{\mathrm{CuCu}}$ a $\mathrm{S}_{\mathrm{NiNi}}$, o $\mathrm{S}_{\mathrm{FeFe}} \times \mathrm{S}_{\mathrm{FeNi}}$. (b) et (c) décomposition des $S_{\mathrm{ij}}(\mathrm{q})$ en $S_{\phi}, S_{\varepsilon}$ selon l'équation (10).

Les facteurs de pondération $\mathrm{X}_{\mathbf{i}}$ sont très voisins de ceux déduits dans le modèle à deux phases et définissent donc la conode; les facteurs $\mathrm{X}_{\mathbf{i}}$ peuvent être assimilé à ceux d'une pseudoconode. Les valeurs de $X_{i}$ et $Y_{i}$ ne dépendent pas du traitement thermique (vieillissement isotherme à 460,500 , et $550^{\circ} \mathrm{C}$ avec une dynamique de taille de domaines variant entre 20 et $50 \mathrm{~nm}$ ). Le profil de $S_{\varepsilon}$ est similaire à celui des $S_{i j}$ ou de $S_{\phi}$ : les fluctuations de concentration contribuant à ce terme supplémentaire ont une longueur de corrélation similaire à celle du motif à deux phases. La description la plus plausible pour $S_{\varepsilon}$, discutée en / 15/, est un enrichissement de Fe aux interfaces cohérents entre $\mathrm{Cu}$ et NiFe.

On a cherché à préciser l'origine de ce terme supplémentaire. La principale particularité du système $\mathrm{Cu} \mathrm{Ni} \mathrm{Fe}$ est l'anisotropie du motif. La morphologie en forme de "tweed pattern" consiste en trois familles de plaquettes selon les plans $\{100\}$, alternativement enrichies en $\mathrm{FeNi}$ et $\mathrm{Cu}$ et empilees selon une des directions $<100>$. 
L'anisotropie de ce motif a été étudiée en transmission par ASAXS sur une lame monocristalline /8/. Les Fig. 9a à 9c montrent la diffusion bidimensionnelle (2d) pour les axes de zone principaux (la diffusion anomale dans ce cas n'avait servi que pour renforcer le contraste). Une perspective schématique de la diffusion 3d est ajointe en Fig. 9d.
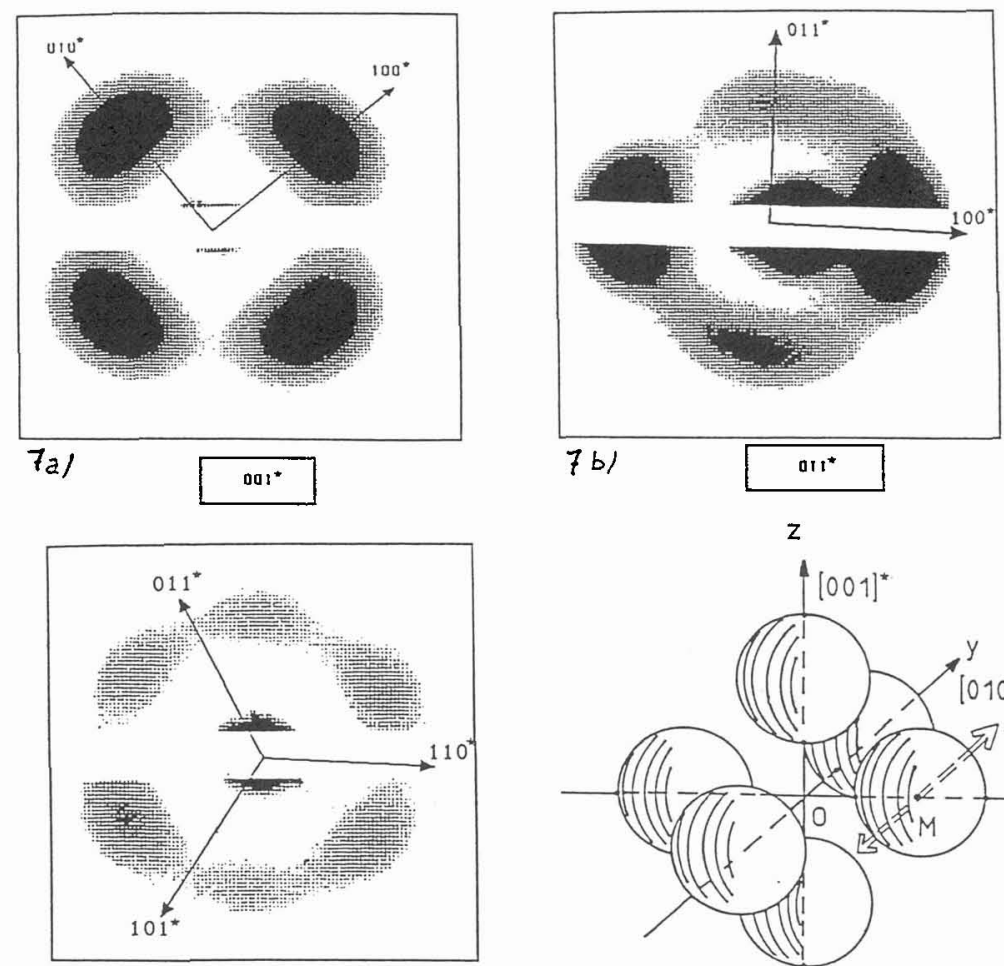

7c)

$111^{*}$

7d)

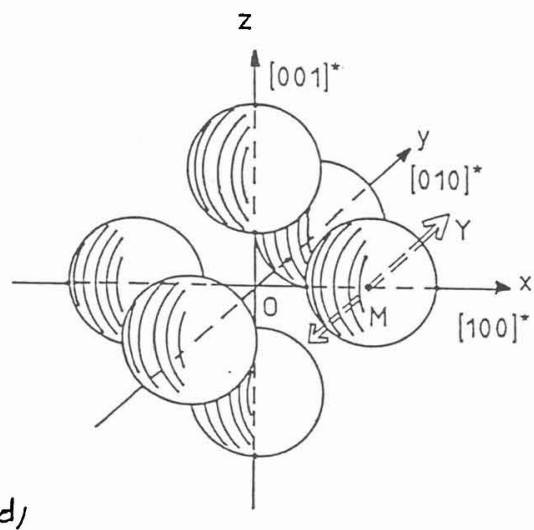

Fig.9 : Photographies de diffusion centrale $2 \mathrm{~d}$ selon les axes de zones indiqués (a, b, c);

représentation schématique de la diffusion $3 \mathrm{~d}(\mathrm{~d})$ où les sphères, centrées sur les maximas d'intensité, simulent la surface d'isointensité à mi-hauteur.

Pour toutes les directions de l'espace réciproque passant par l'origine, il y a un maximum formant un anneau. Ce maximum est relatif, les maxima absolus étant selon $\langle 100\rangle^{*}$ et le "col" le plus bas selon $<111\rangle^{*}$. On ne détecte pas les ordres supérieurs et la modélisation du motif ne doit pas envisager une structure parapériodique. Par contre, cette carte de diffusion $3 \mathrm{~d}$ peut être interprétée par des empilements indépendants $<100>$ de plaquettes $\{100\}$. Des mesures d'anomal quantitatives ont été réalisées pour différentes coupes 1d de l'espace réciproque et les PSFs ont été extraits. Leur ordre de grandeur varie d'un facteur 6 entre $\langle 100\rangle *$ et $\langle 110\rangle^{*}$ mais surtout leur importance relative change avec l'orientation : selon $<100\rangle^{*}, \mathrm{~S}_{\mathrm{NiNi}}$ est plus grand que $\mathrm{S}_{\mathrm{CuCu}}$ et $\mathrm{S}_{\mathrm{FeNi}}$ est fortement négatif. Lorsque la direction de mesure s'éloigne de $<100\rangle^{*}, S_{\mathrm{CuCu}}$ devient supérieur à $\mathrm{S}_{\mathrm{NiNi}}$ et $\mathrm{S}_{\mathrm{FeNi}}$ passe de négatif à positif. Ces observations excluent un modèle à deux phases où les rapports entre PSFs ne devraient alors pas dépendre de la direction. De plus la relation (9) n'est clairement pas vérifiée. Si on applique la décomposition donnée par l'équation (10), on trouve que $X_{i}$ et $Y_{i}$ ne dépendent pas de la direction. En prenant pour $S_{\phi}$ et $S_{\varepsilon}$ un modèle d'empilement de plaquettes, on est capable de simuler les résultats expérimentaux $2 \mathrm{~d}$. Le résultat le plus inattendu de cette modélisation est que le terme $S_{\varepsilon}$ est plus important que le terme deux phases $S_{\phi}$ (d'un facteur 2). Cela impliquerait une ségrégation prononcée aux interfaces $\{100\}$. 
Ce travail se poursuit par une étude de la cinétique d'évolution de l'anisotropie à l'aide d'un détecteur bidimensionnel : la décomposition est au début isotrope $\left(0,5 \mathrm{~h} \mathrm{à} 500^{\circ} \mathrm{C}\right)$; l'anisotropie apparait dès $3 \mathrm{~h}$ à $500^{\circ} \mathrm{C}$ et se développe progressivement par une augmentation du diamètre des plaquettes beaucoup plus rapide que leur épaississement. Après $66 \mathrm{~h}$ à $550^{\circ} \mathrm{C}$, les maxima selon $100^{*}$ sont très piqués, indiquant une structure presque périodique. L'analyse quantitative des résultats est en cours.

\section{CONCLUSION}

La contribution de la diffusion anomale aux petits angles pour l'étude de la séparation de phases d'alliages ternaires a été illustrée. La technique normale, largement utilisée pour l'étude de la séparation des alliages binaires, est très sensible, quantitative, mais elle n'est pas sélective. En nanoanalyse, le STEM (Met à balayage avec émission froide) n'est quantitatif qu'au dessus de 10-20 nm. En dessous ne subsistent que deux techniques qui nous paraissent complémentaires: la sonde atomique ( cf le papier suivant de Blavette) et l'ASAXS, dont l'échantillonage est macroscopique. L'ASAXS s'applique aussi bien évidemment à d'autres problèmes de transformation de phases, pourvu qu'il y ait formation de particules nanométriques; l'exploitation en est en général plus aisée /1/.

En guise de conclusion, seront données quelques potentialités de l'ASAXS, avec des références à des études en ayant tiré parti, en séparation de phase et en précipitation structurale:

- le renforcement du contraste permet d'étendre le domaine d'application de la diffusion centrale (en particulier aux aciers, où les sources de laboratoires ne donnent pas un contraste suffisant): étude de la précipitation dans le $\mathrm{CuFe} / 16 \%$.

- l'étiquetage d'un élément est important car il permet en général de trancher quant à l'interprétation de la nature des objets diffusants:

interprétation de la queue de diffusion dans les alliages CuNiFe /13/, CuNiSn /17/ ou Invar /18/.

Il permet aussi de séparer la contribution d'un type de particules dans les systèmes multiphasés: en particulier il a permis de montrer que la précipitation structurale dans l'alliage AlLiCuMg consiste en la superposition sans effet croisé de la précipitation de la phase $\mathrm{Al}_{3} \mathrm{Li}$ du système $\mathrm{AlLi}$ et la formation de zones de Guinier Preston de l'alliage $\mathrm{Al}(\mathrm{CuMg}) / 19 /$.

- la détermination des compositions dans des domaines nanométriques:

les autres systèmes étudiés à notre connaissançe par ASAXS sont: CuNiSn /17/, AlZnAg /7,20/, FeNiCoMo /21/ et FeNiW /22/.

- enfin la détermination de facteurs de structure partiels restera l'approche la plus complète, pour caractériser la structure de certains systèmes modèles. Nous avons présenté dans ce papier la démarche expérimentale et les résultats obtenus dans deux systèmes à séparation de phases ternaires. L'alliage Fe Cr Co démixe selon la conode d'équilibre et de ce fait se comporte comme un alliage binaire à substitution isomorphique entre le $\mathrm{Co}$ et le Fe. La cinétique de coalescence en $t^{0,25}$ pourrait être la signature d'une morphologie bipercolee.

Le système $\mathrm{Cu}$ Ni Fe qui à première vue semblait comparable a en fait une morphologie beaucoup plus complexe: d'une part, on ne la peut réduire à une structure biphasée et il y a probablement un enrichissement des interfaces en Fe, d'autre part la coalescence conduit progressivement à une anisotropie100 qui consiste en trois familles de plaquettes $\{100\}$ alternativement riches en $\mathrm{NiFe}$ ou $\mathrm{Cu}$, empilées selon les directions $<100>$. 


\section{REFERENCES}

/1/ SIMON, J.P., O. LYON, Proccedings of the 2nd Conf on Anomalous Scattering, Malente-Hambourg , Aout 92 en publication Elsevier /2/ SIMON, J.P. et O. LYON, Acta Metall. 37 (1989) 1727.

/3/ SIMON, J.P., J.J. HOYT, O. LYON, R. PRO, B.E.C. DAVIS, D. De FONTAINE, J. Appl. Cryst. 18 (1985) 181.

/4/ DUBUISSON, J.M., J.M. DAUVERGNE, C. DEPAUTEX, P. VACHETTE et C.E. WILLIAMS, N.I.M., A246,(1986) 636.

15/ SALVA-GUILARDUCCI, A., J.P. SIMON, P. GUYOT, et I. ANSARA, Acta Metall. 31 (1983) 1705.

/6/ SIMON, J.P., O. LYON et D. de FONTAINE , J. Appl. Crystallog. 18 (1985) 230.

/7/ LYON, O., J.J. HOYT, R. PRO, B.E.C. DAVIS, B. CLARK, D. de FONTAINE et J.P. SIMON, J. Appl. Crystallog. 18 (1985) 480.

/8/ LYON, O. et J.P. SIMON, J. Condens. Matter, 4 (1992) 6073.

19/ GEROLD V., J. Appl. Crystall. 10, (1977) 25.

/10/ ZHU, F, P. HAASEN, R. WAGNER, Acta Metall. 34 (1986) 457.

/11/ WAGNER, W., R. POERSCHKE, H. WOLLENBERGER, "Decomposition in Alloys: the early stages", ed. P. Haasen, (Oxford; Pergamon) (1984) p.170.

/12/ POERSCHKE, R., W. WAGNER, H. WOLLENBERGER et P. FRATZL, J. Phys. F: Metal Phys. 16, (1986) 1905.

/13/ SIMON, J.P. et O. LYON, J. Appl. Crystallog. 24 (1991) 1027.

/14/ LYON O. et J.P. SIMON, Phys. Rev. B35 (1987) 5164.

/15/ LYON O. et J.P. SIMON, J. Phys F: Met. Phys. 18, (1988) 1787.

/16/FRATZL, P, Y. YOSHIDA, G. VOGL et H.G. HAUBOLD, Phys. Rev. B (1993) à publier.

/17/ GOUDEAU, Ph., A. NAUDON et J.M. WELTER, J. Applied Crystall. 23 (1990) 266.

/18/ SIMON, J.P., O. LYON, F. FAUDOT, L. BOULANGER, O. DIMITROV, Acta Metall. 40 (1992) 2693.

/19/ GOMIERO, P., F. LIVET, O. LYON et J.P. SIMON, Acta Metall. 39 (1991) 3007.

/20/ LYON, O.et J.P. SIMON, Acta Metall. 34 (1986) 1197.

121/ BOUZID, N., C. SERVANT et O. LYON, Phil. Mag. B57 (1988) 343.

/22/ SERVANT, C., O. LYON et J.P. SIMON, Acta Metall. 37 (1989) 2403. 